\title{
Acute localized exanthematous pustulosis: A novel side effect of piroxicam
}

\section{Soukaina Maghfour ${ }^{1}$, Monia Youssef ${ }^{1}$, Rim Hadhri $^{2}$, Ines Lahouel', Yosra Soua ${ }^{1}$, Mouna Korbi' ${ }^{1}$, Hichem Belhadjali' ${ }^{1}$, Jameleddine Zili'}

${ }^{1}$ CHU Fattouma Bourguiba Hospital, Department of Dermatology, Monastir, Tunisia, ${ }^{2} \mathrm{CHU}$ Fattouma Bourguiba Hospital, Department of Pathology, Monastir, Tunisia

Corresponding author: Soukaina Maghfour, MD, E-mail: laamranisoukaina28@gmail.com

\begin{abstract}
Acute generalized exanthematous pustulosis (AGEP) is a rare yet well-known cutaneous reaction pattern, mostly caused by drugs. Acute localized exanthematous pustulosis (ALEP) is a localized variant of AGEP. A 42-year-old female presented with multiple erythematous pustules on the face, which appeared three days after the intramuscular injection of piroxicam. Histopathology revealed subcorneal pustules, epidermal spongiosis, and mixed inflammatory cell infiltration in the dermis. The pustules resolved within several days once the patient had discontinued the drug. Herein, we report the first case, as far as we know, of a female with a cutaneous drug reaction consistent with ALEP caused by piroxicam.
\end{abstract}

Key words: Acute localized exanthematous pustulosis; Piroxicam; Nonsteroidal anti-inflammatory drugs; Oxicam

\section{INTRODUCTION}

Acute localized exanthematous pustulosis (ALEP) is a localized variant of acute generalized exanthematous pustulosis (AGEP), which is characterized by the eruption of multiple scattered pustules following drug administration. Antibiotics, mainly $\beta$-lactams and macrolides, is implicated in the majority of cases. Non-steroidal anti-inflammatory drugs (NSAIDs) are also implicated [1]. Nevertheless, there have been no reported cases of ALEP caused by the oxicam family in the literature. Herein, we report the first case of ALEP induced by piroxicam.

\section{CASE REPORT}

A 42-year-old female consulted our dermatology department for the acute onset of facial swelling associated with a burning sensation. A dermatological examination revealed the presence of multiple small, non-follicular pustules on edematous erythema affecting only the face and neck (Figs. la and lb). There was no mucous membrane or nail involvement. The patient had no complaints, especially no fever or general status alteration. She had no personal or family history of dermatological diseases. This pustular eruption developed three days after the intramuscular injection of $20 \mathrm{mg}$ of piroxicam for gonalgia. There was no history of other drug intake, nor infection, nor exposition to other external factors such as mercury. Laboratory investigations revealed a white blood cell count of $10,000 / \mathrm{mm}^{3}$ with a normal differential. There was no eosinophilia. The rest of the biochemical and hematological investigations were within normal limits. Microbiology revealed sterile pustules. Histology revealed a subcorneal pustule associated with epidermal spongiosis (Fig. lc). The superficial dermis was edematous with a prominent perivascular infiltrate of lymphocytes, neutrophils, and eosinophils. According to the EuroSCAR scoring, most of the criteria were met

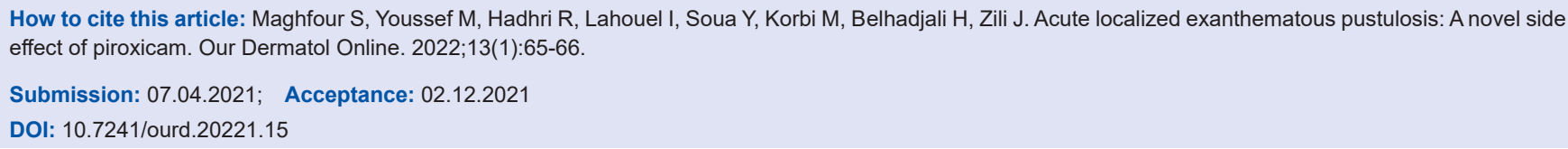




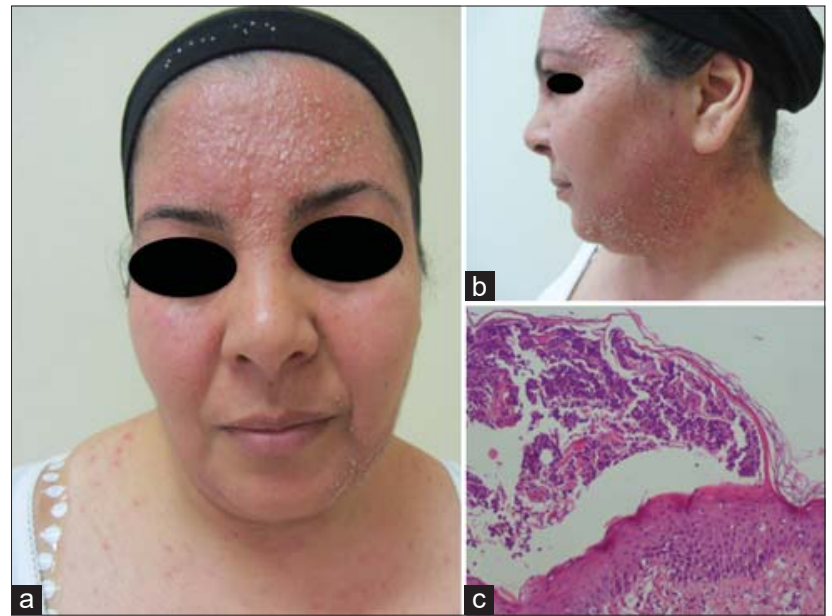

Figure 1: (a-b) Multiple small, non-follicular pustules on edematous erythema affecting the face. (c) Subcorneal pustular with epidermal spongiosis (H\&E, 100x).

by our patient, who had a score of nine, and therefore the case was classified as definite ALEP. Piroxicam was ceased with a resolution of the pustules within several days after topical corticosteroid treatment with desonide cream, followed by characteristic post pustular pinpoint desquamation. The patient refused a follow-up cutaneous patch test.

\section{DISCUSSION}

ALEP is a rare variant of AGEP characterized by the acute onset of multiple, $1-2 \mathrm{~mm}$, non-follicular sterile pustules arising on edematous erythema, localized typically in the face, neck, or chest [2]. Skin symptoms may be associated with fever and leukocytosis. Mucous membranes are rarely affected, commonly mild and limited to one site, mostly the oral lips [1]. Lesions usually resolve rapidly, within several days, after the withdrawal of the causative agent. At this point, a characteristic collaret-shaped post-pustular desquamation may still be a clue to the diagnosis.

ALEP is an uncommon cutaneous drug reaction caused mainly by antibiotics [1]. However, other triggering factors such as bacterial, viral, or parasitic infections, herbal medications, PUVA, mercury, lacquer, venoms, foods, and xenobiotics have also been reported to be involved [3]. ALEP caused by non-steroidal anti-inflammatory drugs (NSAIDs) has been reported only three times in the literature, in three patients, two female and one male, aged 64, 40, and 29, respectively. These cases were induced by ibuprofen, flurbiprofen, and diclofenac, each and respectively [1]. The oxicam family of NSAIDs has not been reported to induce ALEP. The only three compounds of this family that have been implicated in acute exanthematous pustulosis in its generalized form are meloxicam, lornoxicam, and piroxicam [4]. Herein, we report the first case of ALEP induced by piroxicam. Our patient displayed the classic features of ALEP with a characteristic morphology, histology, and course. Although patch testing was not done in our patient, the causative effect of piroxicam was certain due to the course of the affection (the acute onset and the improvement after the withdrawal of the drug). A patch test is an alternative proving the role of a suspected drug and is particularly useful if there may be several causative drugs. Although its sensitivity is around $50 \%$ and negative tests do not allow a final conclusion, positive results are of great value [5].

\section{CONCLUSION}

Piroxicam is a widely prescribed molecule. We report our case to highlight a novel side effect of this drug, which is ALEP, and to increase awareness of this side effect among clinicians.

\section{Consent}

The examination of the patient was conducted according to the principles of the Declaration of Helsinki.

The authors certify that they have obtained all appropriate patient consent forms, in which the patients gave their consent for images and other clinical information to be included in the journal. The patients understand that their names and initials will not be published and due effort will be made to conceal their identity, but that anonymity cannot be guaranteed.

\section{REFERENCES}

1. Villani A, Baldo A, De Fata Salvatores G, Desiato V, Ayala F, Donadio C. Acute localized exanthematous pustulosis (ALEP): Review of literature with report of case caused by amoxicillinclavulanic acid. Dermatol Ther (Heidelb). 2017;7:563-70.

2. Mohamed M, Soua Y, Njim L, Hammemi S, Youssef M, Akkari H, et al. [Acute localized exanthematous pustulosis on the face: 6 cases in Tunisia]. Ann Dermatol Venereol. 2014;141:756-64.

3. Szatkowski J, Schwartz RA. Acute generalized exanthematous pustulosis (AGEP): A review and update. J Am Acad Dermatol. 2015;73:843-8.

4. Larif S, Slim R, Fathallah N, Ghariani N, Hmouda H, Ben Salem C. Lornoxicam-induced acute generalized exanthematous pustulosis. Int J Dermatol. 2016;55:e458-60.

5. Altaykan A, Boztepe G, Erkin G, Özkaya Ö, Özden E. Acute generalized exanthematous pustulosis induced by bleomycin and confirmed by patch testing. J Dermatol Treat. 2004;15:231-4.

Copyright by Soukaina Maghfour, et al. This is an open access article distributed under the terms of the Creative Commons Attribution License, which permits unrestricted use, distribution, and reproduction in any

medium, provided the original author and source are credited.

Source of Support: Nil, Conflict of Interest: None declared. 